\title{
Knife crime: the volunteer doctors teaching lifesaving skills to teenagers
}

We have corrected this Feature article (BMJ 2019;367:16363, doi:10.1136/bmj.16363) to make it clear that Simon Jackson and Nick Rhead's initial exposure to the extent of young people's experiences of violence was through their volunteering at a local youth offending team, not in A\&E. 